\title{
A Matrix with Real Characteristic Roots
}

\section{Karl Goldberg}

\begin{abstract}
It is proved that a certain matrix, which is the coefficient matrix of a differential equation found in the theory of dielectric relaxation, has only real characteristic roots. This is done by finding a real symmetric matrix with the same principal minors and thus the same characteristic roots.
\end{abstract}

In papers $[1,2,3]^{1}$ by J. D. Hoffman and B. M. Axilrod a certain differential equation has a real, constant coefficient matrix $A=\left(a_{i j}\right)$ with the properties

$$
\begin{array}{cl}
a_{i i} \geq 0 & \text { for all } i \\
a_{i j} \leq 0 & \text { for all } i \neq j \\
\sum_{i} a_{i j}=0 & \text { for all } j \\
a_{i_{1} i_{2}} a_{i_{2} i_{3}} \ldots a_{i_{k} i_{1}}= & =a_{i_{2} i_{1}} a_{i_{3} i_{2}} \ldots a_{i_{1} i_{k}} \\
& \text { for all }{ }_{i_{1}, i_{2}}, \ldots, i_{i_{k}} .
\end{array}
$$

In this note we prove the conjecture of $\mathrm{J}$. D. Hoffman and B. M. Axilrod that a matrix with these properties has only real characteristic roots and that these characteristic roots lie between zero and twice the maximum diagonal element.

The second statement is a direct consequence of the first and properties (1), (2), and (3) by the theorem of S. Gershgorin [4] and A. Brauer [5]. This theorem states that all the characteristic roots of a matrix $A=\left(a_{i j}\right)$ lie in the area bounded by the circles $\left|z-a_{j j}\right| \leq \sum_{i}\left|a_{i j}\right|-\left|a_{j j}\right|$.

Therefore, we may concentrate on proving that all the characteristic roots are real. We shall do this by exhibiting a symmetric matrix with the same characteristic roots as one with properties (2) and (4).

Let $B=\left(b_{i j}\right)$ be a matrix of the same order as the matrix $A=\left(a_{i j}\right)$, which has properties (2) and (4), such that

$$
b_{i j}=-\left(a_{i j} a_{j i}\right)^{1 / 2}
$$

with the square root assumed to be positive. Then

$$
\begin{aligned}
b_{i_{1} i_{2}} b_{i_{2} i_{2}} \ldots b_{i_{k} i_{1}} & \\
= & (-1)^{k}\left(a_{i_{1} i_{2}} a_{i_{2} i_{1}} a_{i_{2} i_{3}} a_{i_{3} i_{2}} \ldots a_{i_{k} i_{1}} a_{i_{1} i_{k}}\right)^{1 / 2} \\
& =(-1)^{k}\left|a_{i_{1} i_{2}} a_{i_{2} i_{3}} \ldots a_{i_{k} i_{1}}\right| \\
& =a_{i_{1} i_{2}} a_{i_{2} i_{3}} \ldots a_{i_{k} i_{1}}
\end{aligned}
$$

for all $i_{1}, i_{2} \ldots, i_{k}$

\footnotetext{
1 Figures in brackets indicate the literature references at the end of this paper.
}

Now consider any principal minor

$$
\left|A\left(s_{1}, s_{2}, \ldots, s_{m}\right)\right|=\left|a_{i j}\right|_{i, j=s_{1}, s_{2}}, \ldots, s_{m} \text { of } A .
$$

From the definition of a determinant we have

$$
\left|A\left(s_{1}, s_{2}, \ldots, s_{m}\right)\right|=\sum \pm a_{s_{1} t_{1}} a_{s_{2} t_{2}} \ldots a_{s_{m} t_{m}}
$$

where the sum is taken over all permutations

$$
\left(\begin{array}{ccc}
s_{1} s_{2} & \ldots & s_{m} \\
t_{1} t_{2} & \ldots & t_{m}
\end{array}\right)
$$

of $s_{1}, s_{2}, \ldots, s_{m}$, and the sign is positive if the permutation is even, and negative if it is odd.

Each permutation is the product of cycles. Thus

$$
\left(\begin{array}{cccc}
s_{1} s_{2} & \ldots & s_{m} \\
t_{1} t_{2} & \ldots & t_{m}
\end{array}\right)=\left(\begin{array}{ccc}
i_{1} i_{2} & & i_{k} \\
i_{2} i_{3} & \cdots & i_{1}
\end{array}\right) \ldots,
$$

so that

$$
\begin{aligned}
a_{s_{1} t_{1}} a_{s_{2} t_{2}} \ldots a_{s_{m} t m} & =a_{i_{1} i_{2}} a_{i_{2} i_{3}} \ldots a_{i_{k} i_{1}} \ldots \\
& =b_{i_{1} i_{2}} b_{i_{2} i_{3}} \ldots b_{i_{k} i_{1}} \ldots \\
& =b_{s_{1} t_{1}} b_{s_{2} t_{2}} \ldots b_{s_{m} t_{m}} .
\end{aligned}
$$

It follows that

$$
\left|A\left(s_{1}, s_{2} \ldots, s_{m}\right)\right|=\left|B\left(s_{1}, s_{2}, \ldots, s_{m}\right)\right|,
$$

that is, that the corresponding principal minors of $A$ and $B$ are equal.

This implies that $A$ and $B$ have the same characteristic equation and thus have the same characteristic roots.

[1] J. D. Hoffman and B. M. Axilrod, Dielectric relaxation for spherical molecules in a crystalline field: Theory for two simple models, J. Research NBS 54, 375 (1955) RP2598.

[2] J. D. Hoffman, Theory of dielectric relaxation for a single-axis rotator in a crystalline field. II, J. Chem. Phys. 23, 1331 (1955).

[3] B. M. Axilrod, Dielectric relaxation for a three-dimensional rotator in a crystalline field: Theory for a general six-site model, J. Research NBS 56, 81 (1956) RP2651.

[4] S. Gershgorin, Über die Abgrenzung Engenwerte einer Matrix, Izvest. Akad. Nauk, S.S.S.R. 7, 749 (1931).

[5] A. Brauer, Limits for the characteristic roots of a matrix, Duke Math. J. 13, 387 (1946).

Washington, October 20, 1955. 